\title{
Comparative Finite Element Model Analysis of Ascending Aortic Flow in Bicuspid and Tricuspid Aortic Valve
}

\author{
$*$ Francesca Viscardi, $†$ Christian Vergara, $\neq$ Luca Antiga, $¥$ Sabrina Merelli, \\ $\S$ Alessandro Veneziani, *Giovanni Puppini, *Giuseppe Faggian, *Alessandro Mazzucco, \\ and *Giovanni Battista Luciani \\ *Divisions of Cardiac Surgery and Radiology, University of Verona, Verona; $†$ Department of Information Technology and \\ Mathematical Methods, University of Bergamo, $\neq$ Biomedical Engineering Department, Mario Negri Institute, Bergamo, \\ Italy; and §Department of Mathematics and Computer Science, Emory University, Atlanta, GA, USA
}

\begin{abstract}
In bicuspid aortic valve (BAV) disease, the role of genetic and hemodynamic factors influencing ascending aortic pathology is controversial. To test the effect of BAV geometry on ascending aortic flow, a finite element analysis was undertaken. A surface model of aortic root and ascending aorta was obtained from magnetic resonance images of patients with BAV and tricuspid aortic valve using segmentation facilities of the image processing code Vascular Modeling Toolkit (developed at Mario Negri Institute). Analytical models of bicuspid (antero-posterior [AP], type 1 and latero-lateral, type 2 commissures) and tricuspid orifices were mathematically defined and turned into a volumetric mesh of linear tetrahedra for computational fluid dynamics simulations. Numerical simulations were performed with the finite element code LifeV. Flow velocity fields were assessed for four levels: aortic annulus, sinus of Valsalva, sinotubular junction, and ascending aorta. Comparison of finite element analysis of bicuspid and tricuspid aortic valve showed different blood flow velocity pattern.
\end{abstract}

Flow in bicuspid configurations showed asymmetrical distribution of velocity field toward the convexity of midascending aorta returning symmetrical in distal ascending aorta. On the contrary, tricuspid flow was symmetrical in each aortic segment. Comparing type $1 \mathrm{BAV}$ with type 2 $\mathrm{BAV}$, more pronounced recirculation zones were noticed in the least. Finally, we found that in both BAV configurations, maximum wall shear stress is highly localized at the convex portion of the mid-ascending aorta level. Comparison between models showed asymmetrical and higher flow velocity in bicuspid models, in particular in the AP configuration. Asymmetry was more pronounced at the aortic level known to be more exposed to aneurysm formation in bicuspid patients. This supports the hypothesis that hemodynamic factors may contribute to ascending aortic pathology in this subset of patients. Key Words: Bicuspid aortic valve-Ascending aorta-Finite element modelHemodynamics.
Bicuspid aortic valve (BAV) is the most common form of congenital heart disease, affecting $0.5-2 \%$ of the population (1). It includes different morphological phenotypes, and predisposes to aortic valvar pathology (stenosis, regurgitation, or both) and aortic aneurysms at different levels, even in children and

doi:10.1111/j.1525-1594.2009.00989.x

Received May 2009; revised November 2009.

Address correspondence and reprint requests to Giovanni Battista Luciani, Division of Cardiac Surgery, University of Verona, O. C. M. Piazzale Stefani 1, Verona, 37126, Italy. E-mail: giovanni. luciani@univr.it

Read at the 5th International Conference on Pediatric Mechanical Support Systems and Pediatric Cardiopulmonary Perfusion, Dallas, TX, May 27-30, 2009. young adults, irrespective to severity of valvar dysfunction (2). The pathogenesis of aortic dilation in the presence of BAV is still controversial. Histopathologic changes as cystic medial necrosis of the proximal aortic wall causing abnormal aortic distensibility and stiffness were identified in patients with BAV, not differently from Marfan patients (3). A genetic basis accounting for both valve and wall defects was thus postulated (BAV syndrome). However, unlike patients with Marfan syndrome, patients with BAV do not suffer from pulmonary artery dilatation, countering the idea of an inherited tissue weakness (1). In addition, recent studies have shown that a variety of genotypes is associated with the BAV phenotype (4). Another possible 
explanation for aortic aneurysms in BAV patients is a pathophysiological phenomenon due to increased wall stress caused by abnormal blood flow in the aortic root through a stenotic BAV. Nonetheless, aortic dilation is noted also in patients with a functionally normal or regurgitant valve. One hypothesis is that the abnormal opening of the BAV, even if not stenotic or only mildly stenotic, may cause increased hemodynamic wall stress leading to aneurysm formation (5).

3 In order to elucidate the role of aortic valve (AV) morphology (bicuspid vs. tricuspid) and orientation (bicuspid with antero-posterior [AP] vs. bicuspid with latero-lateral [LL] commissures) on patterns of ascending aortic flow dynamics, a computational model of the aortic root was constructed. This allows to investigate in a noninvasive and in a quantitative way the blood flow through the AV $(6,7)$. In particular, the aims of this study were to: (i) create computational models of arterial vessels, starting from geometrical data obtained by digitalized magnetic resonance imaging (MRI); (ii)perform numerical simulations in these geometries by the finite element method, which allows the computation of the velocity field and wall shear stress (WSS) of blood in the ascending aorta and evaluation of the subsequent risk for aneurysm formation in a stated site; and (iii) examine qualitative differences in aortic blood flow between tricuspid valvular orifice and bicuspid ones.

\section{METHODS}

\section{Patients and cardiac imaging}

Institutional Review Board approval was obtained for the conduct of this study, and the board waived the need for patient consent. Six healthy subjects (aged 16-65 years) with an incidental trans-thoracic echocardiographic (TTE) finding of BAV and eight with a finding of tricuspid aortic valve (TAV) underwent cardiac MRI. TTE was used to detect AV anatomy and to exclude relevant (moderate or greater) valve insufficiency and relevant (peak gradient $>20 \mathrm{~mm} \mathrm{Hg}$ ) aortic stenosis. AV morphology was examined in parasternal long- and short-axis views. A BAV was diagnosed when two cusps were clearly identified in short-axis view. AP BAV, hereafter named type $1 \mathrm{BAV}$, was defined by the presence of ideal fusion of right and left coronary cusps, while LL $\mathrm{AV}$, hereafter named type $2 \mathrm{BAV}$, when right coronary and noncoronary cusps were fused. Left ventricular outflow tract measurements, including ascending aorta, were carried out in two-dimensional parasternal long-axis approach at four levels (annulus, sinuses of Valsalva, sinotubular junction,

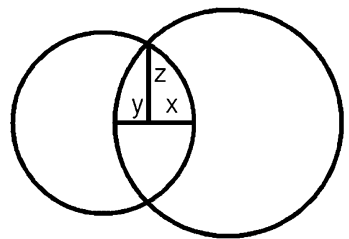

FIG. 1. Intersection of two circle functions of different radii in order to determine the different valves. Tricuspid valves correspond to $x=y=z=9.8 \mathrm{~mm}$, and bicuspid ones to $x=4, y=6$, $z=9.8$.

and proximal ascending aorta), perpendicular to the axis of the aorta at each level. Peak aortic velocity, and peak and mean aortic gradients were assessed using the continuous-wave Doppler technique from different imaging planes. Doppler imaging was applied to measure the deceleration slope and pressure half-time of the aortic regurgitant jet. Transthoracic echocardiogram was performed with 2.5$\mathrm{MHz}$ ultrasound transducers (Hewlett-Packard Sonos 500 system, $\bullet \bullet$ ) and recorded on VHS videotape. MRI was obtained in vivo by a 1.5 Tesla machine (Magnetom Simphony, Siemens Medical Systems, Erlangen, Germany). Spin-echo sequences for morphological definition were obtained from the cardiac base to the aortic arch. For a multiphase imaging of the AV sequences, K-space turbo gradient echo (TrueFisp) was used, acquired during a 12-s breath hold for each view with retrogated ECG triggering, set acquisition window $20 \%$ above the average $\mathrm{R}-\mathrm{R}$ interval. The following parameters were used: $\mathrm{TE}=1.6 \mathrm{~ms}$; flip angle $=65^{\circ}$; slice thickness $=6 \mathrm{~mm}$; temporal resolution $=48 \mathrm{~ms}$; field of view $=400 \mathrm{~mm}$; acquisition matrix $=256 \times 256$. From the short-axis plane, left ventricle outflow-tract cine sequences were acquired, and ascending aortic flow was evaluated through cine gradient-echo images, highlighting its relation with the geometry of the opening of aortic leaflets.

\section{Construction of the meshes from MRI data}

A surface model of the aortic root, ascending aorta, aortic arch, and thoracic aorta of one of the six subjects was obtained from MRI images using the segmentation facilities of an image processing research code, the Vascular Modeling Toolkit (8). In particular, this tool allows the generation of a surface representing the lumen boundary located at the steepest lumen intensity change. An analytical model of a bicuspid valve orifice was mathematically defined on a two-dimensional plane by the intersection of two circle functions of different radii (Fig. 1). This function was sampled on the surface represent- 


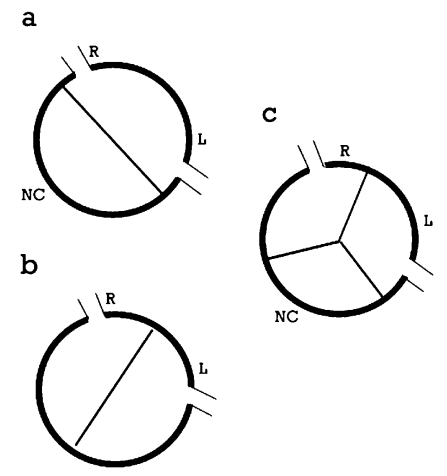

FIG. 2. Schematic classification of BAV phenotypes with respect to TAV in an orientation similar to echocardiographic parasternal short-axis view: (a) type 1 BAV (fusion of left and right coronary cusps); (b) type 2 BAV (fusion of right coronary and noncoronary cusps); (c) TAV. R, $\bullet$; L, $\bullet$; NC, $\bullet$.

ing the aortic root inlet and was used to open an orifice resembling a typical bicuspid valve with given parameters AV area, AV orientation, position of the valve inside aorta. This was done for type 1 and type 2 BAV configurations as well as for the tricuspid valve model. In particular, the area of the root was of $4.6 \mathrm{~cm}^{2}$; the area of the tricuspid valve was $3.2 \mathrm{~cm}^{2}$ (corresponding to $\mathrm{x}=\mathrm{y}=\mathrm{z}=9.8 \mathrm{~mm}$ in Fig. 1); and the area of the bicuspid valves was $2.0 \mathrm{~cm}^{2}$ (corresponding to $\mathrm{x}=4, \mathrm{y}=6, \mathrm{z}=9.8 \mathrm{~mm}$ in Fig. 1), according to available information on normal and diseased AV anatomy $(9,10)$. The orientation of the two bicuspid valve configurations was set according to the respective commissures (Fig. 2), with relative angles equal to $65^{\circ}$. The solid models were successively turned into volumetric meshes of linear tetrahedra in order for computational fluid-dynamics simulations to be carried out (Fig. 3). Mesh generation has been performed using the Vascular Modeling Toolkit, as described in reference (8): the quality of the surface mesh is first improved and its density adapted according to the user specification, and the final surface volume is then tetrahedralized by means of the

11 Tetgen mesh generation library (8). In all the three meshes, we have about 1300000 tetrahedra. This dimension was reached after successive mesh refinements, with the aim of obtaining a mesh-independent numerical solution. An example of the relation between patient MRI and modeling is reported in Fig. 4.

\section{Numerical simulations}

Unsteady and laminar numerical simulations were performed in these computational domains with the finite element code LifeV (a library jointly developed at the research centers MOX-Politecnico di Milano,

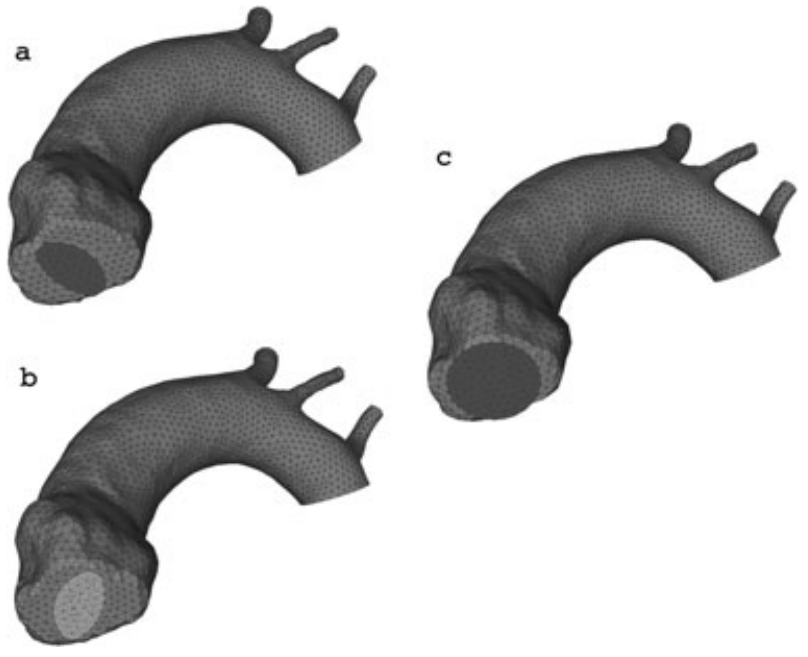

FIG. 3. Mesh representing ascending aorta. The three valve orifice models are also presented: (a) type 1 BAV; (b) type 2 BAV; (c) TAV.

INRIA-Paris, CMCS-EPFL-Lausanne, and more recently at the University of Bergamo and at the Emory University-see http://www.lifev.org). The blood was considered as Newtonian, homogeneous, and incompressible, so that the Navier-Stokes equa-

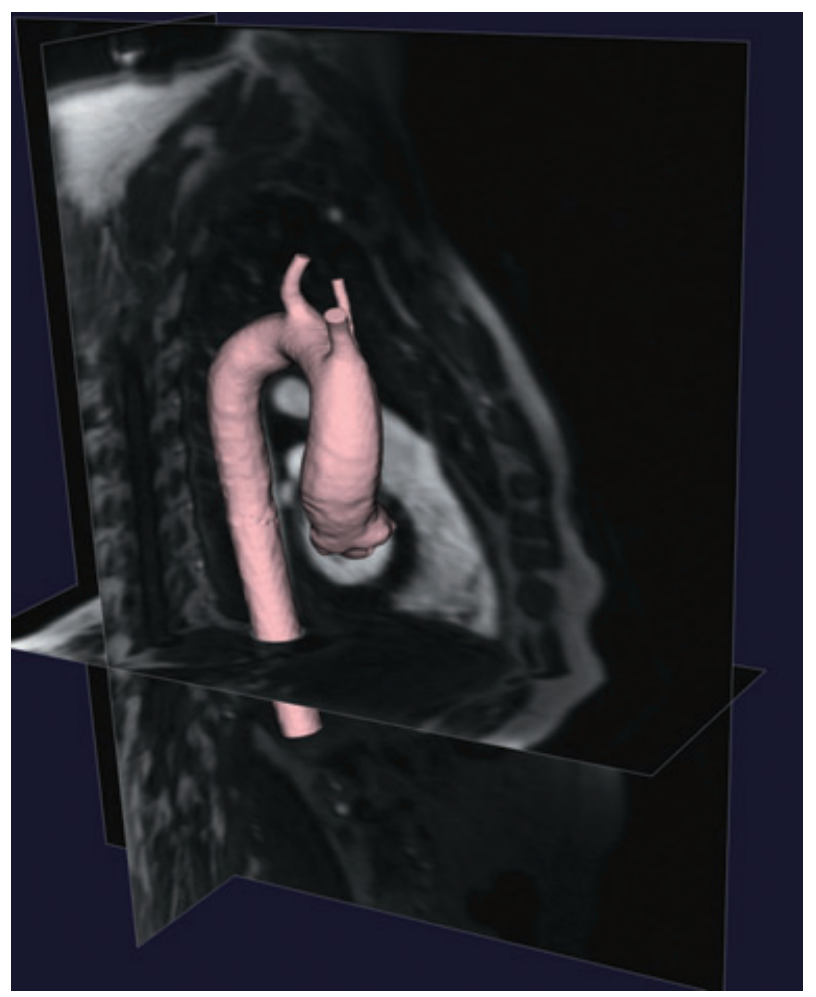

FIG. 4. Example of the relationship between patient MRI and 20 completed vascular modeling process. 


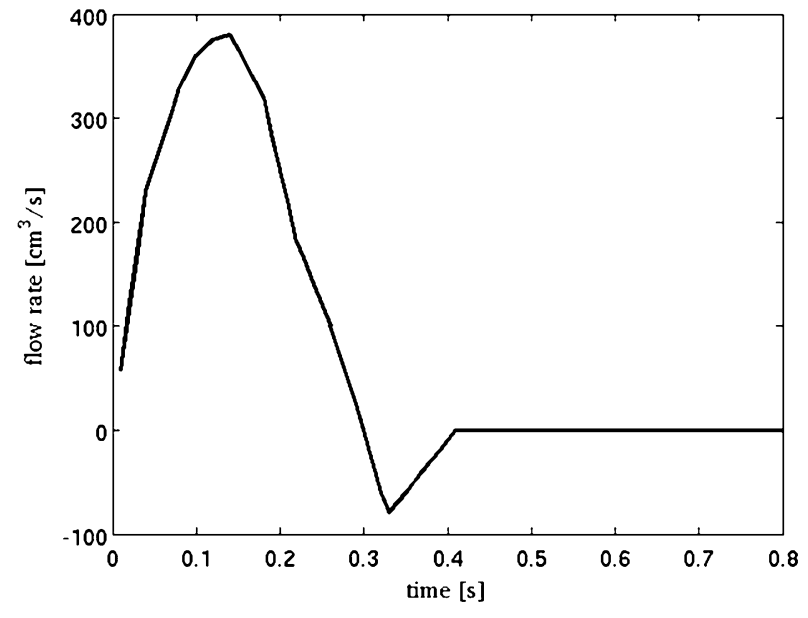

FIG. 5. Flow rate boundary condition prescribed at the inlet of the ascending aorta as representative of the heart action (taken from (8)).

tions for incompressible fluids were used for the mathematical description (11). These assumptions are known to be valid for blood flowing into large and medium vessels. The fluid viscosity was set equal to $0.035 \mathrm{P}$ and the density equal to $1.0 \mathrm{~g} / \mathrm{cm}^{3}$. We used a time discretization parameter equal to $0.008 \mathrm{~s}$. We resorted to a parallel implementation of our finite element solver, exploiting a 48-processor architecture. The vessel wall was considered rigid (fixed) and the geometry corresponded to the systolic ejection phase of the cardiac cycle. The valve opening and closure were modeled in an on/off modality. In particular, the valve was open for the first $0.4 \mathrm{~s}$ of the heartbeat and closed for the remaining time (Fig. 5).

At the inlet, physiological inflow boundary conditions (Fig. 5) taken from were chosen as representa-

13 tive of the heart action (12). To this aim, a specific nonstandard mathematical technique was used to avoid any bias introduced by choosing a priori the shape of the velocity profile at the inlet, as commonly done in this context $(13,14)$. The implementation of such a technique in LifeV and the numerical simulations presented in these work have been performed at the University of Bergamo.

\section{RESULTS}

The numerical results obtained by the finite element method concern the velocity and the pressure of the blood in the reconstructed geometries. We point out that the velocity profile at the inlet computed with our approach was quite different from the (constant) profile that one would obtain if a blunt profile was assumed. In particular, the ratio between the maximum velocity at the systole computed by our solver and the value of the blunt profile, which would fit the flow rate at that instant, was equal to 2.88 for type $1 \mathrm{BAV}$ and 2.18 for the tricuspid valve.

The velocity pattern at the early systole (time = $0.096 \mathrm{~s}$ ) was assessed at four different aortic levels: (i) aortic annulus, (ii) sinus of Valsalva, (iii) sinotubular junction, and (iv) mid-ascending aorta. Vectors of the velocity field obtained for both BAV and TAV configurations are plotted in Fig. 6. In these figures, in order to highlight the differences among the three flow patterns (type 1 and type 2 BAV, TAV), the same velocity range in the bar plot was maintained. The velocity patterns of aortic flow in bicuspid models gain a peak velocity at the systole twofold greater than in the tricuspid model $(5.0 \mathrm{~m} / \mathrm{s}$ for type 1 configuration, $5.2 \mathrm{~m} / \mathrm{s}$ for type 2 configuration, $2.3 \mathrm{~m} / \mathrm{s}$ in TAV). Furthermore, comparison between the two different bicuspid configurations highlights that flow in the type 1 configuration is characterized by a greater asymmetry than in type 2. In Fig. 7, the vectors of the velocity field at time $t=0.216 \mathrm{~s}$ are plotted on the same longitudinal section; this instant
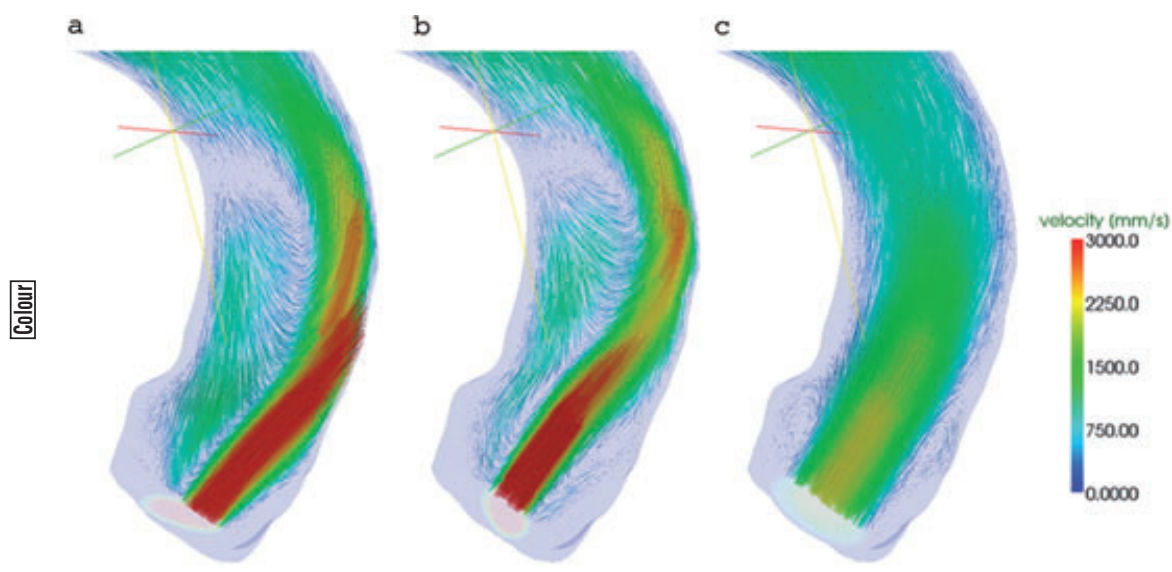

FIG. 6. Vectors of the velocity field plotted in a longitudinal section at time $t=0.098 \mathrm{~s}$ (early systole). On each of the selected points, a vector with length proportional to the magnitude of the velocity field and with the same direction of the field is plotted. The three valve models are presented: (a) type 1 BAV; (b) type 2 BAV; (c) TAV. Greater flow asymmetry is seen in (a) then in (b), while (c) shows no flow asymmetry. 

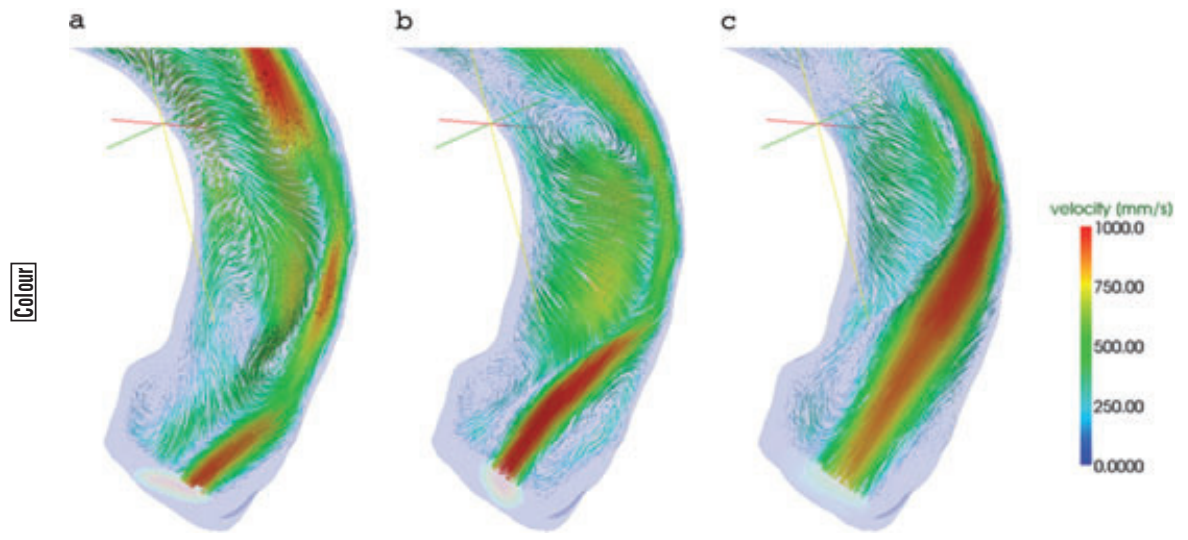

FIG. 7. Vectors of the velocity field plotted in a longitudinal section at time $t=0.216 \mathrm{~s}$ (late systole). On each of the selected points, a vector with length proportional to the magnitude of the velocity field and with the same direction of the field is plotted. The three valve models are presented: (a) type 1 BAV; (b) type 2 BAV; (c) TAV. Again, flow asymmetry is maximum in (a), while recirculation zones are evident in (c).

was chosen to highlight recirculation zones within aortic segments.

Moreover, we show in Fig. 8 the WSS obtained in the three cases, which highlights again the asymmetry of the jet in the bicuspid valve configurations. More precisely, the maximum WSS for BAV configurations is localized around the mid-ascending aorta level, highlighting the big effect of the jet on this portion of the wall.

Finally, we have observed that the degree of vorticity is increased in all cases after systole; in particular, it is maximum at instant $t=0.144 \mathrm{~s}$.

\section{DISCUSSION}

Mathematical and numerical modeling has been widely used to predict arterial aneurysm formation, even at the ascending aortic level (15). The application of computational modeling with finite element analysis to solve the clinical question of aortic aneurysm formation relative to BAV represents a novel approach because to our knowledge no prior work on this exists in the literature. As such, several methodological assumptions were necessary to define the "model." Each one of these represents a potential limitation to the model (see Limitations below).
Because of these restrictions, the data generated by these preliminary models are this far only qualitative. Nonetheless, some information is interestingly consistent with previously reported clinical findings. In fact, some authors have pointed out that BAV phenotype can be correlated with severity and localization of aortic aneurysm formation. Histopathologic studies on diseased ascending aortas by Russo et al. (16) state that type $1 \mathrm{BAV}$ is associated with earlier aneurysm formation and worse aortic wall degeneration than type $2 \mathrm{BAV}$ in surgical patients. Echocardiographic findings by Schaefer et al. (17) confirm that type $1 \mathrm{BAV}$ is characterized by a higher abnormal aortic distensibility and stiffness than type 2, with a larger aortic diameter at the sinuses of Valsalva and smaller aortic arch. Further work by Schaefer and associates (18) led to observation that type $1 \mathrm{BAV}$ is associated with normal aortic shape but with greater aortic dimensions, while type 2 is associated with abnormal aortic shape, ascending aortic dilatation, and larger arch dimensions, possibly related to the majority of stenotic valves in the type 2 group. We found in BAV configurations an asymmetrical distribution of velocity field toward the convexity of the mid-ascending aorta, returning symmetrical in the distal ascending aorta. For type $2 \mathrm{BAV}$, the asymme-
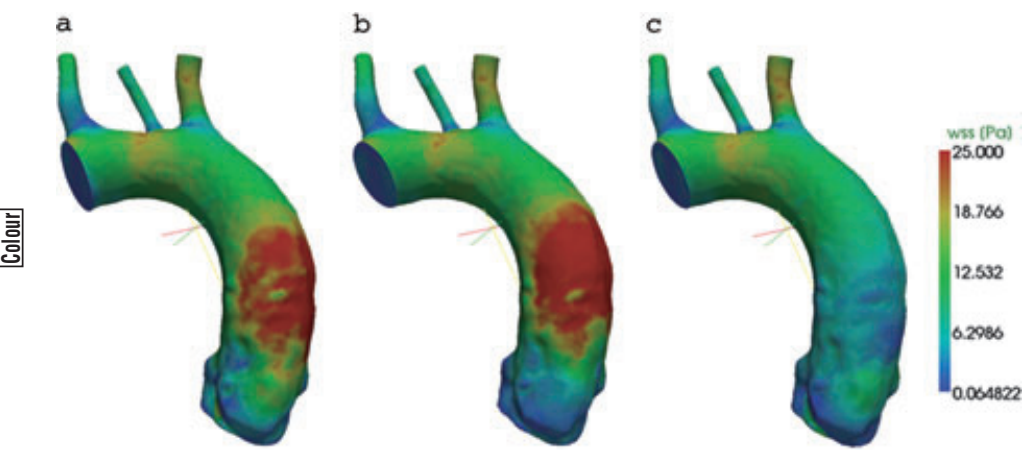

FIG. 8. WSS is plotted. The three valve models are presented: (a) type $1 \mathrm{BAV}$; (b) type $2 \mathrm{BAV}$; (c) TAV. The precise localization of maximum WSS for BAV configurations (corresponding to the red area) is similarly plotted at the convexity of the mid-ascending aorta. 
try is evident but less pronounced with respect to type $1 \mathrm{BAV}$, while recirculation zones and eddy currents are more pronounced and jet velocity is higher, especially at the sinuses of the Valsalva level (Fig. 7). It is likely that the orientation of the BAV openings with respect to the plane of aortic curvature results in different jet shapes and different distribution of wall stress on the aorta. In the type 1 model, BAV configuration is oriented less symmetrically with respect to the plane of aortic curvature, which leads to a stronger jet oriented toward the outer wall of the ascending aorta. This is confirmed also by the distribution of the maximum WSS, which is highly localized in both BAV configurations. Different WSS

14 could lead to vascular remodeling and aneurysm formation as observed in BAV patients, which is associated not only with the severity and mechanism of valve dysfunction but mainly with valve morphology and orientation.

Discrepancies, if any, between clinical (echocardiographic) observations (16-18) and the present numerical results are to be ascribed to the fact that most clinical series include diseased (to a varying extent) valves, mostly regurgitant. In this work, the aortic orifice was set at an area compatible with normal (i.e., nonstenotic) valve function $(9,10)$. This choice was deliberately done to distinguish the influence of AV geometry on aortic flow pattern and, ultimately, on asymmetrical stress on the entire ascending aorta. It is noteworthy that the few clinical series where morphological subtypes of BAV were correlated with aortic dimensions, in the absence of a significant valve dysfunction (normally functioning BAV), failed to demonstrate any association (18). Indeed, BAV has thus far been thought to cause dilatation only due to stenotic orifice. Some authors $(5,19)$ have found that in stenotic BAV patients, the anterolateral region of the ascending aorta is subject to greater hemodynamic stress (measured with Doppler flow velocity) than in stenotic TAV patients. The present study offers preliminary evidence that even normally functioning BAV (i.e., nonstenotic) may generate flow patterns and velocity identical to those seen in poststenotic hemodynamics (and dilatation).

Our preliminary conclusion is that there is something inherent with aortic flow modeled by a twoleaflet valve that is intrinsically different (thus pathological) from three-leaflet valve flow. Echocardiographic works pointing out that, irrespective to the functional status of the valve, BAV is usually associated with a predominant enlargement of midascending aorta support these findings (19). In fact, in our study, mid-ascending aortic level is the one with maximum flow velocity and more pronounced jet asymmetry, and maximum WSS, especially in type 1 BAV. In addition, nonstenotic BAV with different orientation (and analyzed here are the two comprising the vast majority of theoretically infinite configurations) produce different levels and severity, and thus morphological types, of aortic aneurysm. The present work suggests that the methodology applied here allows the generation of mathematical models for computational fluid dynamics starting from clinical data (echocardiography, MRI). In addition, such a method may enable solution by abstraction of specific clinical questions (i.e., does number and orientation of AV leaflets generate different flow patterns and fluid dynamics?) and, ultimately, allow return to the clinical setting with predictive information.

\section{LIMITATIONS OF THE STUDY}

There are a few notable limitations to this study. First of all, the vessel wall was considered fixed. This assumption is not realistic for the aorta, but, due to its simplicity, this model was chosen to give insightful preliminary results. The study of the compliant model, which is more complex from the computational point of view, is under further investigation.

Second, the orientation, shape, and area of the valves have been chosen as representative of the three different anatomic types, based on echocardiographic findings recorded from individuals thought to be representative of that BAV phenotype, as previously done by others (16). This was a simplification, because, as clinicians and surgeons know, each BAV patient carries a slightly different valvular anatomy and function and aortic dimension. Furthermore, surgical (and pathological) classifications are often at variance with echocardiographic ones (16-18,20,21). Nevertheless, a simplification is needed for the application of the proposed model. Obviously, the choice of different valvar parameters might alter the final results. However, as the aim of this work is to give preliminary results with a chosen methodology, we focused on the two more commonly reported BAV morphologies (AP, type 1, and LL, type 2) in most cardiological and surgical series. The analysis of computational domains with slightly different parameters of the valve (such as area and orientation) is still ongoing and may corroborate these findings.

Another limitation has been introduced in modeling the opening and closure of the valve. In particular, an on/off modality has been chosen. It is worth noting, however, that the dynamics of valve opening are very fast, and we do not expect that they have an influence on the shape and direction of the jet in systole, which is our main observation variable.
55 
Finally, we point out that the results presented herein are purely qualitative, our main purpose being to illustrate the eventual impact of an integrated multidisciplinary approach with some preliminary results. However, the proposed conjectures will need to be supported by quantitative results. For this reason, future work will be focused on the quantification of some fluid-dynamics quantity. For example, it will be possible to consider, together with the WSSs, also the energy levels in order to detect if there is any correlation between the velocity patterns and the aneurysm formation.

\section{CONCLUSIONS}

Comparison between the three models with the proposed finite element analysis shows an asymmetrical and higher flow velocity in the bicuspid models. The asymmetry is different between the two phenotypes: BAV configurations show a higher velocity jet at the aortic level known to be more exposed to aneurysm formation in bicuspid patients. In particular, flow obtained in the type 1 BAV configuration shows a more pronounced asymmetry, while the one in the type $2 \mathrm{BAV}$ configuration shows higher recirculation zones at the sinuses of the Valsalva level. Moreover, the maximum WSS in both BAV configurations is localized at the convexity of the midascending aorta level. All these findings support the hypothesis that hemodynamic factors may contribute to ascending aortic pathology in patients with BAV. They might also support the thesis that attributes differences in aortic dimensions between BAV phenotypes to inhomogeneous distribution of shear forces due to the different relation of leaflet orientation with ascending aortic geometry.

\section{REFERENCES}

1. Guntheroth WG. A critical review of the American College of Cardiology/American Heart Association practice guidelines on bicuspid aortic valve with dilated ascending aorta. Am J Cardiol 2008;102:107-10.

2. Gurvitz M, Chang RK, Drant S, Allada V. Frequency of aortic root dilation in children with a bicuspid aortic valve. Am J Cardiol 2004;94:1337-40.

3. Bonderman D, Gharehbaghi-Schnell E, Wollenek G, Maurer G, Baumgartner H, Lang IM. Mechanisms underlying aortic dilatation in congenital aortic valve malformation. Circulation 1999;99:2138-43.

4. Ellison JW, Yagubyan M, Majumdar R, et al. Evidence of genetic locus heterogeneity for familial bicuspid aortic valve. J Surg Res 2007;142:28-31.
5. Bauer M, Siniawski H, Pasic M, Schaumann B, Hetzer R. Different hemodynamic stress of the ascending aorta wall in patients with bicuspid and tricuspid aortic valve. J Card Surg 2006;21:218-20.

6. Pekkan K, Whited B, Kanter K, et al. Patient-specific surgical planning and hemodynamic computational fluid dynamics optimization through free-form haptic anatomy editing tool (SURGEM). Med Biol Eng Comput 2008;46:1139-52.

7. Weinberg EJ, Kaazempur Mofrad MR. A multiscale computational comparison of the bicuspid and tricuspid aortic valves in relation to calcific aortic stenosis. J Biomech 2008;41:3482-7.

8. Antiga L, Piccinelli M, Botti L, Ene-Iordache B, Remuzzi A, Steinman DA. An image-based modeling framework for patient-specific computational hemodynamics. Med Biol Eng Comput 2008;46:1097-112.

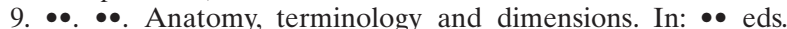
Kirklin/Barratt-Boyes. Cardiac Surgery, 3rd Edition. New

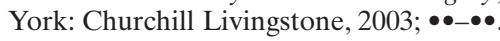

10. American College of Cardiology/American Heart Association Task Force on Practice Guidelines, Society of Cardiovascular Anesthesiologists, Society for Cardiovascular Angiography and Interventions, et al. ACC/AHA 2006 guidelines for the management of patients with valvular heart disease: a report of the American College of Cardiology/American Heart Association Task Force on Practice Guidelines (writing committee to revise the 1998 Guidelines for the Management of Patients With Valvular Heart Disease): developed in collaboration with the Society of Cardiovascular Anesthesiologists: endorsed by the Society for Cardiovascular Angiography and Interventions and the Society of Thoracic Surgeons. Circulation 2006;114: e84-231.

11. Formaggia L, Quarteroni A, Veneziani A, eds. Cardiovascular Mathematics. $\bullet$ : Springer, 2009.

12. Avolio AP. Multi-branched model of the human arterial system. Med Biol Eng Comp 1980;18:709-18.

13. Formaggia L, Gerbeau JF, Nobile F, Quarteroni A. Numerical treatment of defective boundary conditions for the NavierStokes equation. SIAM J Numer Anal 2002;40-1:376-401.

14. Veneziani A, Vergara C. Flow rate defective boundary conditions in haemodinamics simulations. Int J Numer Meth Fluids 2005;47:803-16.

15. Poullis MP, Warwick R, Oo A, Poole RJ. Ascending aortic curvature as an independent risk factor for type A dissection, and ascending aortic aneurysm formation: a mathematical model. Eur J Cardiothorac Surg 2008;33:995-1001.

16. Russo CF, Cannata A, Lanfranconi M, Vitali E, Garatti A, Bonacina E. Is aortic wall degeneration related to bicuspid aortic valve anatomy in patients with valvular disease? J Thorac Cardiovasc Surg 2008;136:937-42.

17. Schaefer BM, Lewin MB, Stout KK, Byers PH, Otto CM. Usefulness of bicuspid aortic valve phenotype to predict elastic properties of the ascending aorta. Am J Cardiol 2007; 99:686-90.

18. Schaefer BM, Lewin MB, Stout KK, et al. The bicuspid aortic valve: an integrated phenotypic classification of leaflet morphology and aortic root shape. Heart 2008;94:1634-8.

19. Cecconi M, Manfrin M, Moraca A, et al. Aortic dimensions in patients with bicuspid aortic valve without significant valve dysfunction. Am J Cardiol 2005;95:292-4.

20. Della Corte A, Romano G, Tizzano F, et al. Echocardiographic anatomy of ascending aorta dilatation: correlations with aortic valve morphology and function. Int $J$ Cardiol 2006;113:320-6.

21. Sievers HH, Schmidtke C. A classification system for the bicuspid aortic valve from 304 surgical specimens. J Thorac Cardiovasc Surg 2007;133:1226-33. 


\begin{tabular}{|l|l|}
\hline \multicolumn{2}{|c|}{ Toppan Best-set Premedia Limited } \\
\hline Journal Code: AOR & Proofreader: Emily \\
\hline Article No: 989 & Delivery date: 6 January 2010 \\
\hline Page Extent: 7 & Copyeditor: Sharon \\
\hline
\end{tabular}

\section{AUTHOR QUERY FORM}

Dear Author,

During the preparation of your manuscript for publication, the questions listed below have arisen. Please attend to these matters and return this form with your proof.

Many thanks for your assistance.

\begin{tabular}{|c|c|c|}
\hline $\begin{array}{l}\text { Query } \\
\text { References }\end{array}$ & Query & Remark \\
\hline 1 & $\begin{array}{l}\text { AUTHOR: Please check and confirm that the city location for University of } \\
\text { Verona is correct. }\end{array}$ & \\
\hline 2 & $\begin{array}{l}\text { AUTHOR: Journal style requires the Abstract to be unstructured. Please check } \\
\text { and confirm that the rewriting of the abstract is OK. }\end{array}$ & \\
\hline 3 & $\begin{array}{l}\text { AUTHOR: AV: Is this the correct abbreviation of aortic valve? Please note that } \\
\text { subsequent mentions of aortic valve has been changed to AV. Please confirm } \\
\text { this is correct. Please also note that the abbreviation AV has appeared in the } \\
\text { text (e.g., latero-lateral [LL] AV ) as per original manuscript. Thus, if AV has a } \\
\text { different definition please make the necessary changes in the text. }\end{array}$ & \\
\hline 4 & $\begin{array}{l}\text { AUTHOR: Trans-thoracic echocardiogram: Should this be abbreviated as TTE } \\
\text { as well? }\end{array}$ & \\
\hline 5 & $\begin{array}{l}\text { AUTHOR: Please provide the manufacturer details (company, city, state [if } \\
\text { applicable], country) for Hewlett-Packard Sonos } 500 \text { system. }\end{array}$ & \\
\hline 6 & $\begin{array}{l}\text { AUTHOR: Is TrueFisp a commercial name? If so, please provide the manufac- } \\
\text { turer details (company, city, state [if applicable], country). }\end{array}$ & \\
\hline 7 & $\begin{array}{l}\text { AUTHOR: R-R interval: should R-R be spelled out? If so, please provide its } \\
\text { full form. }\end{array}$ & \\
\hline 8 & AUTHOR: TE: should this be spelled out? If so, please provide its full form. & \\
\hline 9 & $\begin{array}{l}\text { AUTHOR: Vascular Modeling Toolkit: Please provide manufacturer/developer } \\
\text { details (company, city, state [if applicable], country). }\end{array}$ & \\
\hline 10 & $\begin{array}{l}\text { AUTHOR: "This was done for type } 1 \text { and type } 2 \ldots . \text { ": This sentence has been } \\
\text { reworded for clarity. Please check and confirm it is correct. }\end{array}$ & \\
\hline 11 & $\begin{array}{l}\text { AUTHOR: Tetgen: Please provide manufacturing details (company, city, state } \\
\text { [if applicable], country). }\end{array}$ & \\
\hline 12 & $\begin{array}{l}\text { AUTHOR: MOX, INRIA, CMCS, EPFL: Are these company names? Company } \\
\text { names don't need to be spelled out per journal style. Otherwise, please spell } \\
\text { out MOX, INRIA, CMCS, EPFL. }\end{array}$ & \\
\hline
\end{tabular}




\begin{tabular}{|c|c|}
\hline 13 & $\begin{array}{l}\text { AUTHOR: At the inlet, physiological inflow boundary conditions (Fig. 5) taken } \\
\text { from were chosen as representative of the heart action: A word seems to be } \\
\text { lacking in this sentence. Please reword or confirm it is correct. }\end{array}$ \\
\hline 14 & $\begin{array}{l}\text { AUTHOR: "Different WSS ...": this sentence has been reworded for clarity. } \\
\text { Please check and confirm it is correct. }\end{array}$ \\
\hline 15 & $\begin{array}{l}\text { AUTHOR: "In addition, such ...": This sentence has been reworded for } \\
\text { clarity. Please check and confirm it is correct. }\end{array}$ \\
\hline 16 & $\begin{array}{l}\text { AUTHOR: Reference 9: This has been assumed to be a chapter in a book refer- } \\
\text { ence entry. Please provide author name/s, editor name/s and page range. }\end{array}$ \\
\hline 17 & AUTHOR: Please provide the location of publisher in Reference 11. \\
\hline 18 & $\begin{array}{l}\text { AUTHOR: Reference } 13 \text { and Reference 14: Please check and confirm that } \\
\text { abbreviated journal titles are correct. }\end{array}$ \\
\hline 19 & AUTHOR: FIG. 2: Please define L, R, and NC. \\
\hline 20 & $\begin{array}{l}\text { AUTHOR: Please send a completed color charge form to the production editor } \\
\text { at AOR@ bos.blackwellpublishing.com if you wish to have your figures printed } \\
\text { in color. The color charge form can be found on the e-proofing website. If a } \\
\text { form is not sent, your figures will be converted to black and white. If you have } \\
\text { already sent a completed form, then please ignore this query. Thank you. }\end{array}$ \\
\hline
\end{tabular}

